\section{Ethical commitments}

WHO Constitution (1946): "...the highest attainable standard of health as a fundamental right of every human being"

WHO Sustainable Development Goals (2015) No. 3.8: Achieve universal health coverage, induding financial risk protection, access to quality essential healthcare services and access to safe, effective, quality and affordable essential medicines and vaccines for all."

EU Charter of Fundamental Rights (2012) Art-35: "Everyone has the right of access to preventive health care and the right to benefit from medical treatment under the conditions extablished by national laws and practices:"

\section{Economic considerations}

Can societies afford equitable healthcare for all? Should nation states allow access to a full range of services also for people who reside without authorization on their territory? Or should irregular migrants' access be restricted to emergency care, as is common in most European countries?

Resolution 1946 (2013) - Parliamentary Assembly - Council of Europe on Equal Access to Health Care:

3. [..] inequalities in access to care, induding mental health care, particularly affect vulnerable groups, [o. ] especially those in an irregular situation [...]. These inequalities lead to a phenomenon of non-recourse or delayed recourse to care, which could have disastrous implications for both individual and public health and lead in the long term to an increase in health expenditure."

\section{Evidence on costs of care}

Using different methodological approaches and data sources, recent studies come to similar conclusions: it is not cost saving to restrict access to health care to emergency treatment.

An economic model to calculate costs for two medical conditions - hypertension and prenatal care - was applied to three EU Member States: Germany, Greece and Sweden. It indicated that providing access to regullar preventive healthcare for migrants in an irregular situation would be cost-saving for healthcare systems.

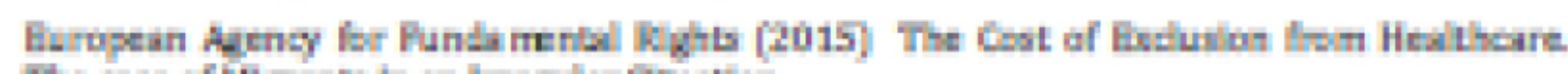
The ase of Migrants in in lregular Sivition.

A quasi-experimental study in Germany using data from 1994-2013 demonstrated that restrictions on asylum seekers' and refugees' healthcare entitlements ultimately incur higher costs than regular access to care.

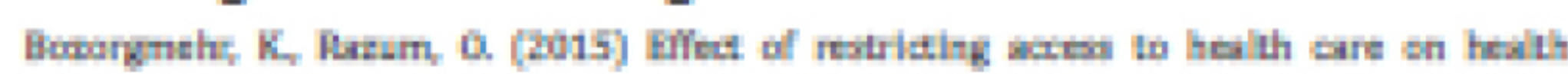

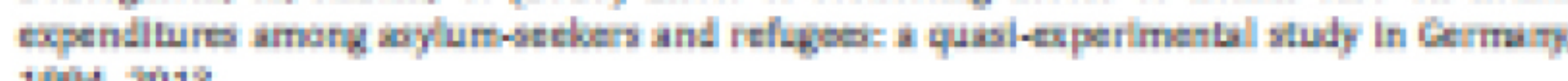
17ititois

A vignette study using a micro-costing approach in four European countries (AT, BE, ES, IT) analysing 6 primary care sensitive medical conditions (asthma, depression, diabetes, epilepsy hyptertension, TB) shows the cost-saving potential of timely treatment in primary care settings.

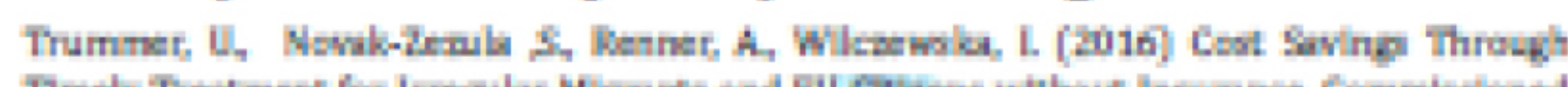

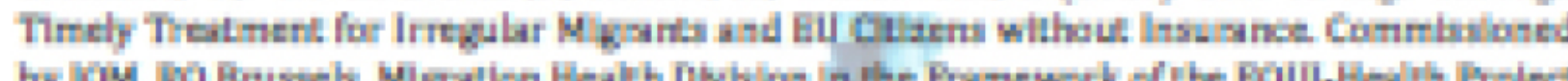

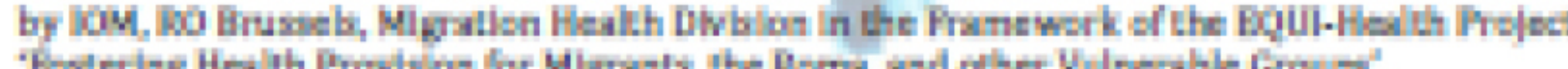

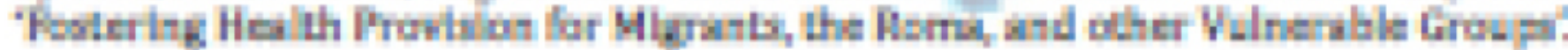

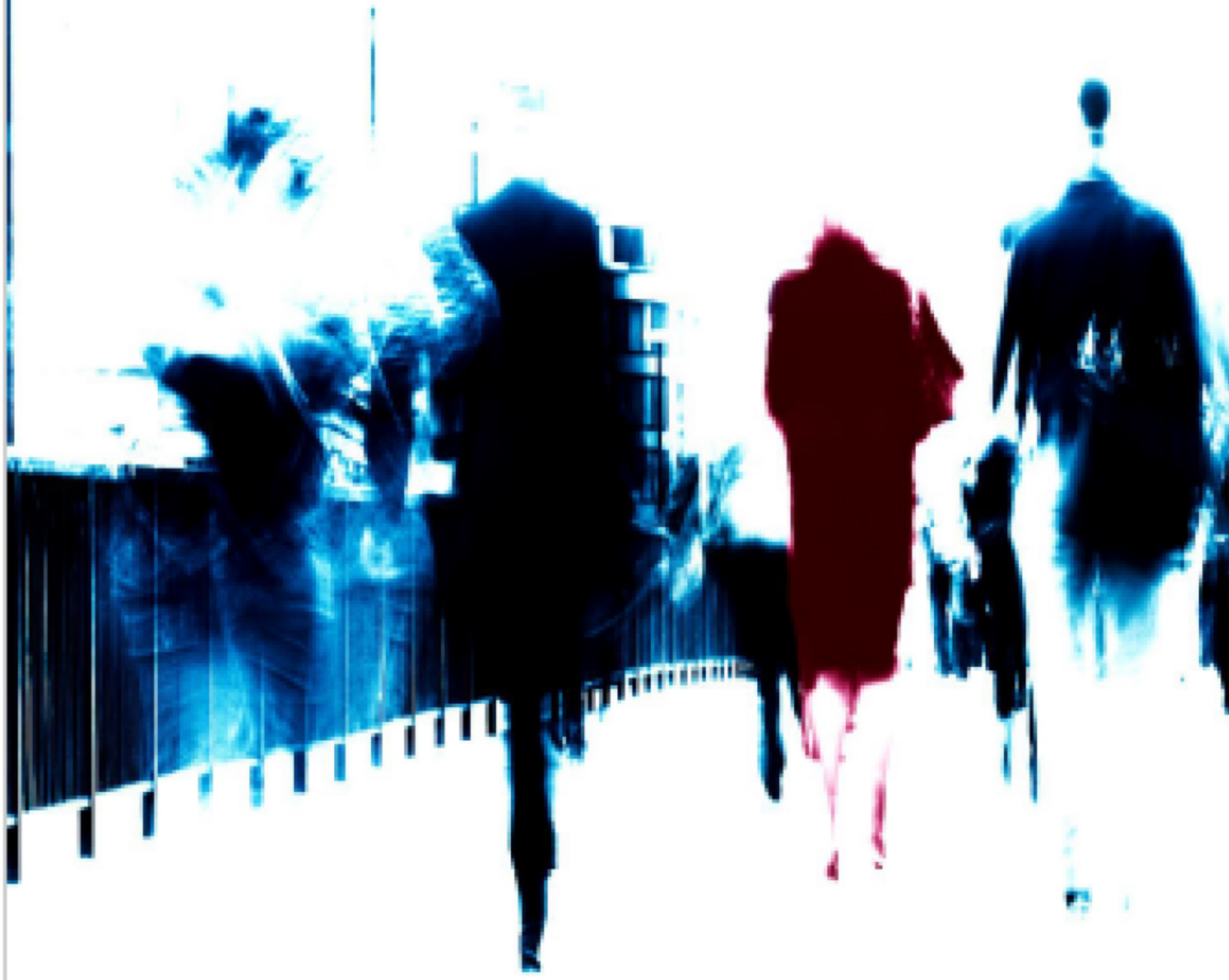

Join the EUPHA working group on economic arguments in migrant health policy-making!

The working group provides a forum for discussions around ethics, economics, and deservingness; e.g. what does ultimately tip the scales in decision-making processes on migrants' health entitlements, value-based or evidence-based ' arguments?

Contact:

the_economic_argument@c-hm.com

office@c-hm.com
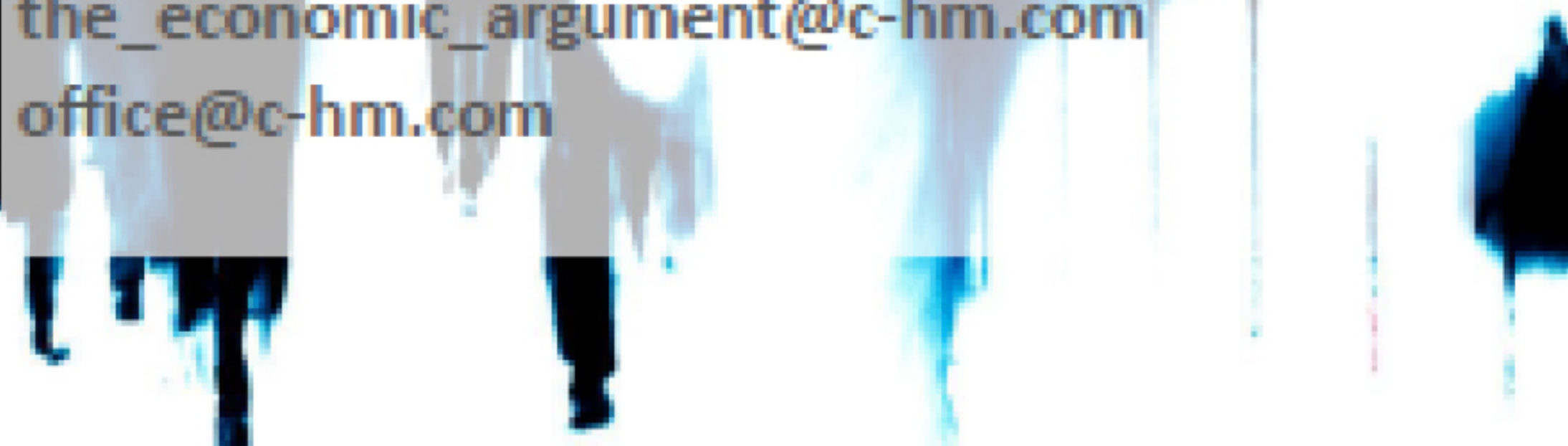

\title{
Ethics \& economics regarding access to healthcare for marginalised groups
}
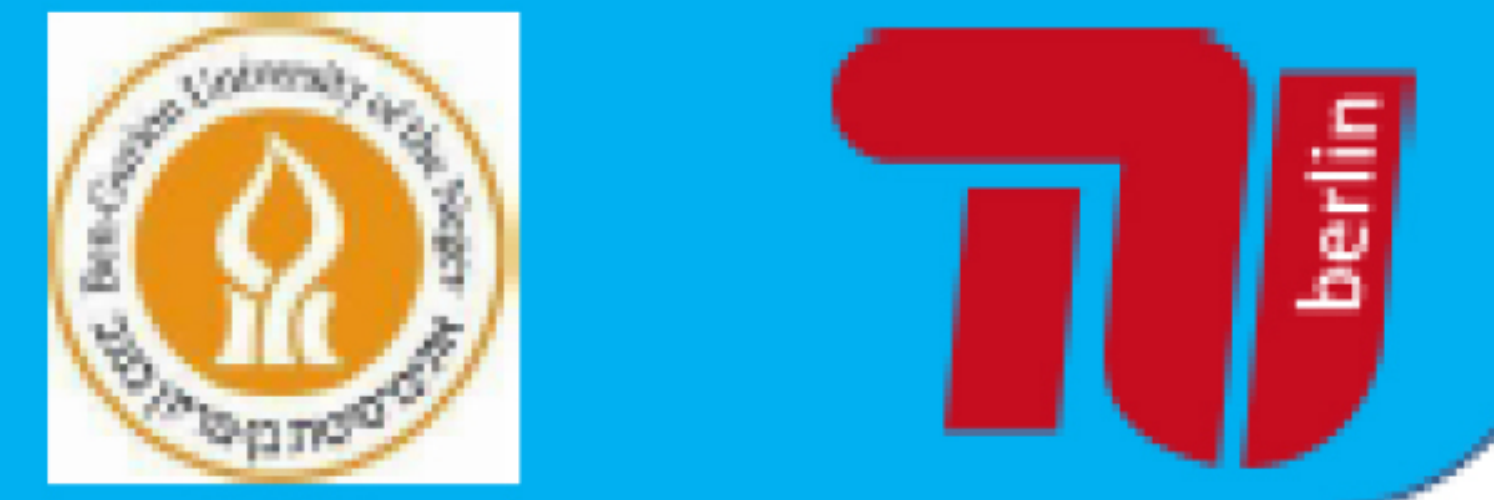